\title{
Scoliosis manager for medical doctors: a new Internet free tool to enhance medical approach to scoliosis worldwide
}

\author{
Stefano Negrini", Claudia Fusco, Fabio Zaina, Alberto Negrini \\ From 7th International Conference on Conservative Management of Spinal Deformities \\ Montreal, Canada. 20-22 May 2010
}

\begin{abstract}
Introduction
Internet is changing health problems management in many perspectives. A new Open Access (OA) Software developed, experimented and used since 6 years in our Institute is going to be freely offered to the international scoliosis MD community (ScoliosisManager - SM http://www.scoliosis.manager.com) and is presented in this paper.
\end{abstract}

\section{Materials and methods}

Since the foundation of our Institute in 2003 we started a Software to allow complete data collection of all our patients. It was developed in Access for Windows; after 2 years it was transferred in Internet; finally, it was converted to web 2.0 and we are experimenting its usage by third parties not involved in our Institute, before launching the free SM worldwide.

\section{Results}

In December 2009 the medical part of SM included 24,822 evaluations of 8,261 patients; it has been used by 11 MDs for all their patients in 21 different Italian locations; each MD freely access to its own patients' information through Internet.

SM includes the following modules (one per patient): anagraphics, personal data, general clinical history, treatment history, clinical diary. Other modules (one per evaluation) are: clinical evaluation (history, observation, TRACE, sagittal plane, ATR and hump, anthropometrics, others), diagnosis and prescription. Finally, there are $\mathrm{x}$-ray modules (12,898 filled in). Since October

ISICO (Italian Scientific Spine Institute), Milan, Italy

Full list of author information is available at the end of the article
2008 we started also photographic dossiers of patients (14,237 photos). SM allows complete printing reports.

SM allows in daily routine to reduce times, have complete data collection and printout for patients and colleagues; the learning curve is rapid and requires around 30 to 40 evaluations to be optimised. Moreover, SM allows complete records for research (29 papers published from this database until now) and quality control purposes.

\section{Discussion}

In 2010 the International collaboration with third parties with the free use of SM will start, to test it and receive suggestions for future advancements. Before the end of 2011 the project will be completed and SM will be totally free in Internet for the general use.

\section{Conclusion}

Internet is going to be the backbone of information flow and communities around the world. Developing an OA (and eventually in the future Open Source) resource for the Scoliosis MD community could allow in the next future developments and improvements we are not yet ready to see in their completeness. Our yearly experience in this particular field is ready to be exported and with this presentation we want to let colleagues fully know and start contributing to this advancement.

Published: 10 September 2010

doi:10.1186/1748-7161-5-S1-042

Cite this article as: Negrini et al:: Scoliosis manager for medical doctors:

a new Internet free tool to enhance medical approach to scoliosis

worldwide. Scoliosis 2010 5(Suppl 1):O42. 